\title{
Differential and Single-Ended Microstrip Lines Loaded with Slotted Magnetic-LC Resonators
}

\author{
J. Naqui, M. Durán-Sindreu, and F. Martín \\ GEMMA/CIMITEC, Departament d'Enginyeria Electrònica, Universitat Autònoma de Barcelona, \\ Bellaterra, 08193 Barcelona, Spain
}

Correspondence should be addressed to F. Martín; ferran.martin@uab.es

Received 28 February 2013; Revised 25 April 2013; Accepted 20 May 2013

Academic Editor: Sha Luo

Copyright (C) 2013 J. Naqui et al. This is an open access article distributed under the Creative Commons Attribution License, which permits unrestricted use, distribution, and reproduction in any medium, provided the original work is properly cited.

This paper is focused on magnetic-LC (MLC) resonators, namely, slotted resonators that can be considered the complementary counterparts of the so-called electric-LC (ELC) resonators. Both resonators exhibit two symmetry planes (i.e., they are bisymmetric), one of them being an electric wall and the other a magnetic wall at the fundamental resonance. Therefore, compared to other electrically small resonators such as folded stepped impedance resonators (SIRs), split ring resonators (SRRs), and their complementary counterparts, MLC and ELC resonators exhibit a very rich phenomenology. In this paper, single-ended microstrip lines and differential microstrip lines loaded with MLC resonators are studied, and potential applications are highlighted.

\section{Introduction}

Split-ring resonators (SRRs) $[1,2]$ and their complementary counterparts (CSRRs) [3] (Figure 1) have been extensively used for the implementation of metamaterials and many devices based on them [4]. SRRs can be excited by means of a uniform axial ( $z$ direction) time-varying magnetic field and/or by an electric field applied in the plane of the particle ( $y$ direction); that is, the particle exhibits bianisotropy [5]. The distribution of charges at the fundamental resonance (see Figure 1(a)) reveals that the symmetry plane of the SRR is an electric wall at that frequency. From duality considerations, it follows that the CSRR can be excited through an axial electric field and/or a magnetic field applied in the $y$ direction, whose symmetry plane behaves as a virtual magnetic wall at the fundamental resonance [6]. Capacitively loaded (C loaded) loops and folded stepped impedance resonators (SIRs), and their complementary counterparts (Figure 2), exhibit a similar phenomenology when they are illuminated with properly polarized radiation to drive the particles.

As long as SRRs, C-loaded loops, folded SIRs, and their dual particles are electrically small, they can be used for the implementation of effective media metamaterials. Specifically, SRRs, C-loaded loops, and folded SIRs are useful for the implementation of negative permeability media following the Lorentz model $[1,7]$, whereas CSRRs, complementary C-loaded loops, and complementary SIRs can be used as building blocks for the implementation of negative permittivity artificial media $[3,8]$. However, resonant-type negative permittivity media can also be achieved by means of metallic particles, as it was pointed out by Schurig and co workers [9]. The particle reported in [9] and depicted in Figure 3 was called electric-LC (ELC) resonator. It was argued that such particle cannot be excited by means of a uniform axial magnetic field since the currents in both loops are opposite at the fundamental resonance; namely, the instantaneous current is clockwise in one of the loops and counterclockwise in the other one. This means that, at the fundamental resonance, the ELC does not exhibit a net magnetic dipole moment in the axial direction, and for such reason it cannot be driven by means of a uniform axial time-varying magnetic field. However, an electric dipole moment appears in the orthogonal direction to the electric wall (indicated in Figure 3), which means that the ELC can be excited through a uniform timevarying electric field applied to that direction. This is the reason that explains the terminology used to designate this particle, which prevents bianisotropy. Notice also that there is another symmetry plane in the particle that acts as a magnetic 


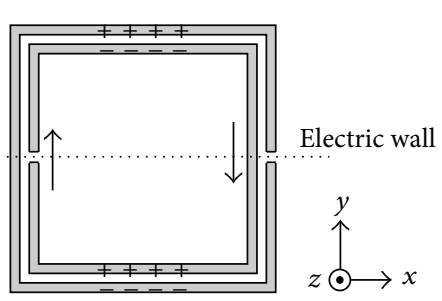

(a)

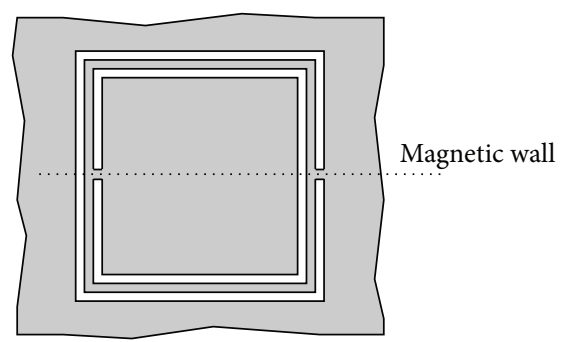

(b)

FIGURE 1: Typical topology of an SRR (a) and a CSRR (b). A sketch of the currents and the distribution of charges for the SRR at the fundamental resonance is also indicated (notice that the symmetry plane is an electric wall). From Babinet's principle, it follows that the CSRR exhibits a magnetic wall at the symmetry plane.

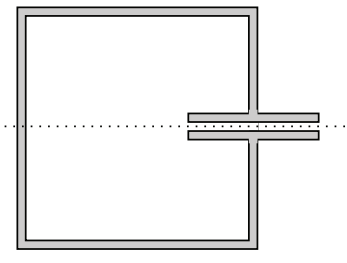

(a)

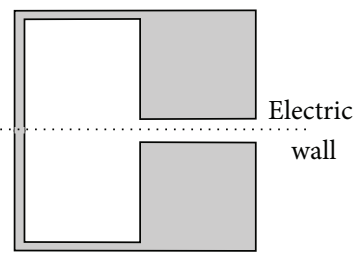

(b)

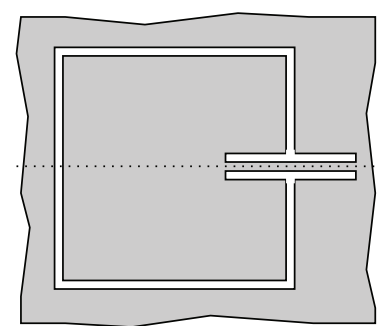

(c)

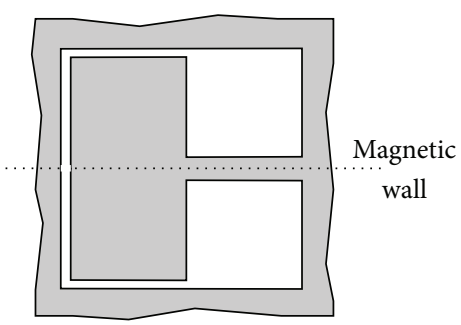

(d)

FIgURE 2: Typical topology of a capacitively loaded loop (a), a folded SIR (b), a complementary capacitively loaded loop (c), and a complementary folded SIR (d).

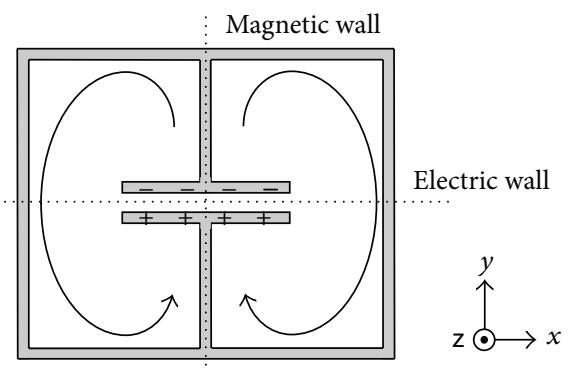

FIGURE 3: Typical topology of an ELC resonator, indicating the electric and magnetic walls and a sketch of the currents and the charge distribution at the fundamental resonance.

wall at the fundamental resonance by virtue of the symmetry in the currents and charges with regard to that plane.

The previous electrically small resonators, including the ELC, can also be used as loading resonant elements in planar transmission lines, where the fields are no longer uniform. In particular, for the ELC, if the driving fields are not uniform, it is also possible to magnetically drive the particle. This can be achieved, for instance, by forcing opposite magnetic flux lines in the loops of the ELC. According to this, the ELC can be a very interesting and useful particle as a loading element in planar transmission lines. However, the main focus in this paper is the study of the dual particle of the ELC, that is, the magnetic-LC (MLC) resonator. This is a slot resonator that can be etched in the ground plane of a single-ended microstrip or a differential microstrip line. The analysis of this particle as well as the potential applications in microstrip configuration, is the main objective of the present work.

\section{Magnetic-LC (MLC) Resonators}

A typical topology of a square-shaped MLC resonator is depicted in Figure 4(a). It is the negative image of the ELC. Hence, by applying the Babinet's principle, it follows that the MLC can be excited by means of a time-varying magnetic field applied in the plane of the particle ( $y$ direction), but not by a uniform electric field normal to the particle plane (which is the usual driving mechanism in CSRRs [3] and in the complementary structures of Figure 2).

Like the ELC, the MLC exhibits two symmetry planes, one being a magnetic wall and the other an electric wall at the fundamental resonance. However, notice that the magnetic and electric walls are rotated $90^{\circ}$ compared to those of the ELC. If the aspect ratio of the slot widths $w_{1} / w_{2}$ is high enough, a quasistatic approximation can be made, and the particle can be described by means of a lumped elementequivalent circuit model, depicted in Figure 4(b). The inductance $L_{m}$ accounts for the inductive path connecting the two inner metallic regions of the particle. This inductance is parallel connected to the capacitances designated as $C_{m}$ that model the slots present between both inner halves of the MLC. Finally, the edge capacitance of the external squareshaped slot ring is called $C_{g}$, and it can be divided into the capacitances of the two MLC halves, as depicted in Figure $4(\mathrm{~b})$. 


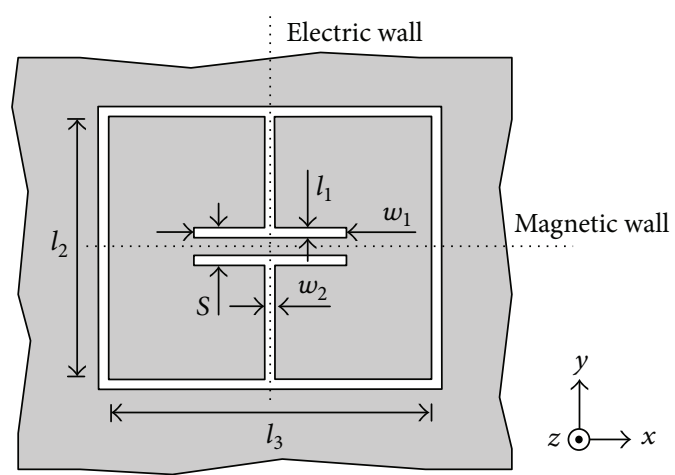

(a)

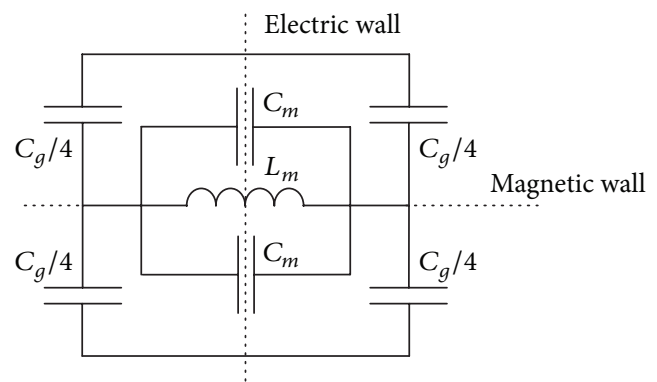

(b)

FIgURE 4: Typical topology of an MLC (a) and its equivalent circuit model (b). The relevant dimensions and the electric and magnetic walls are indicated.

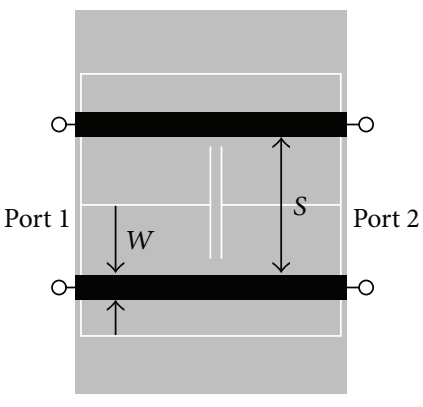

(a)

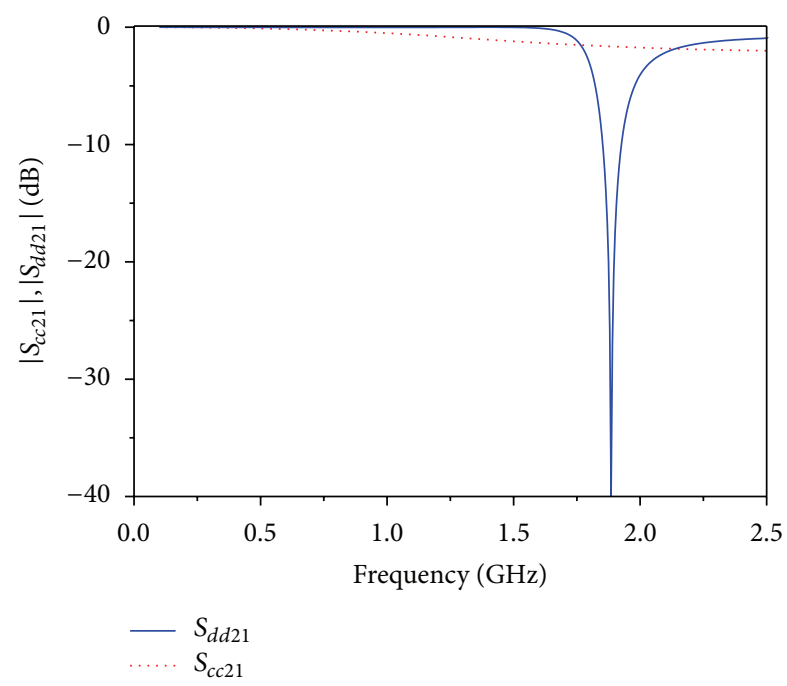

(b)

FIGURE 5: Differential microstrip line loaded with an MLC with the electric wall aligned with the line (a) and frequency response (transmission coefficient) for the common $\left(S_{c c 21}\right)$ and differential $\left(S_{d d 21}\right)$ modes $(\mathrm{b})$. The substrate is Rogers RO3010 with thickness $h=1.27 \mathrm{~mm}$ and dielectric constant $\varepsilon_{r}=11.2$. The line dimensions are $W=1 \mathrm{~mm}$ and $S=5 \mathrm{~mm}$, corresponding to a $50 \Omega$ odd-mode impedance without the presence of the MLC. The MLC dimensions are $w_{1}=4 \mathrm{~mm}, w_{2}=l_{1}=s=0.2 \mathrm{~mm}$, and $l_{2}=l_{3}=10 \mathrm{~mm}$.

Compared to the other complementary structures shown in Figures 1 and 2, which exhibit a magnetic wall at its unique symmetry plane, the MLC exhibits not only a magnetic wall, but also an electric wall, and this additional wall can be useful in certain applications, as it is discussed in the next sections.

\section{Single-Ended Microstrip and Differential Microstrip Lines Loaded with MLCs}

The unusual electric wall that the MLC exhibits at resonance (in comparison to other slotted resonators) opens new research lines. Let us now consider the MLC loading both single-ended microstrip and differential microstrip transmission lines by assuming that the electric wall of the MLC is aligned with the symmetry plane of the lines.
3.1. Differential Microstrip Lines Loaded with MLCs. Figure 5(a) depicts a differential microstrip line loaded with an MLC with the electric wall aligned with the symmetry plane of the line. For the common mode, the symmetry plane of the line is a magnetic wall. Therefore, MLC excitation is not expected for this mode. Conversely, for the differential mode, there is an electric wall at the symmetry plane, and the MLC can be driven. The vertical components of the electric field generated by each individual line under differential mode operation are contra directional (i.e., upwards in one line and downwards in the other line of the pair). Therefore, as long as each inner half of the MLC is below each line of the differential pair, the particle can be excited by the non uniform electric field generated under differential mode excitation. This behavior has been verified through the electromagnetic 


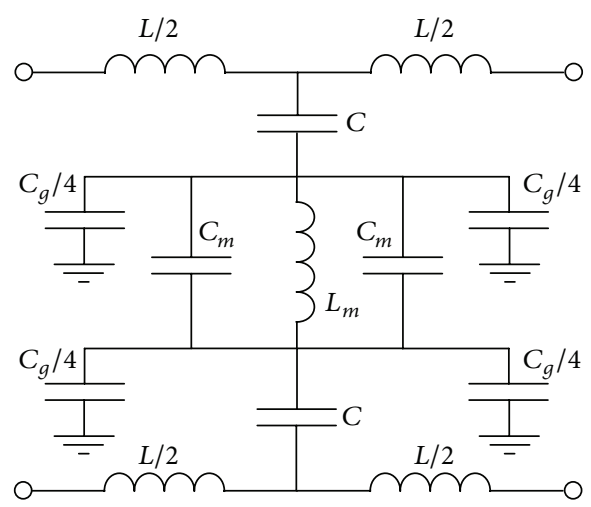

(a)

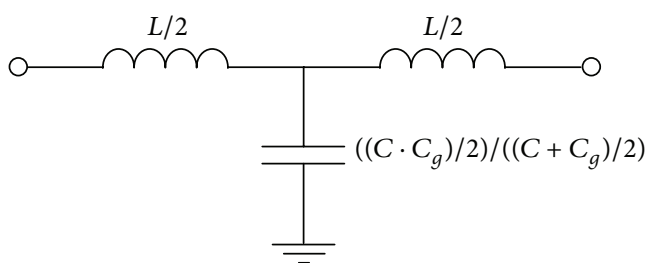

(b)

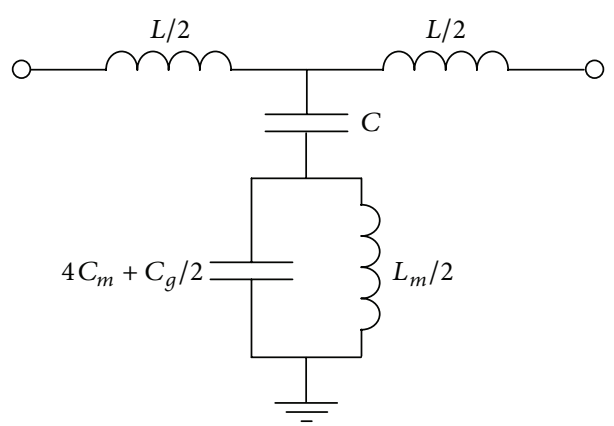

(c)

FIGURE 6: (a) Lumped element-equivalent circuit model of a differential microstrip line loaded with an MLC with the electric wall aligned with the line; (b) circuit model for the even mode, where the symmetry plane is an open circuit; (c) circuit model for the odd mode, the symmetry plane being a short circuit.

simulation of the structure depicted in Figure 5(a) by means of the Agilent Momentum commercial software for both the common and the differential mode (see Figure 5(b)). As expected, the differential line is roughly transparent for the common mode, but it exhibits a notch in the transmission coefficient for the differential mode.

The circuit model of the structure of Figure 5(a) is depicted in Figure 6, where the equivalent circuits for the odd (or differential) and even (or common) modes are also included. The model includes the elements describing the MLC, plus the line inductance $L$ and capacitance $C$. Actually, the former is the line inductance with the presence of the MLC and the later accounts for the electric coupling between the line and the MLC. As it can be appreciated, for the common mode there is no transmission zero even though the shunt capacitance is modified. On the contrary, for the differential mode, the resulting model is the same as that for a CSRR-loaded microstrip line which exhibits a transmission notch [10]. In order to validate the model for the differential mode, we have considered the structure depicted in Figure 7(a) which is electrically smaller at resonance than that in Figure 5(a) mainly by virtue of the higher aspect ratio $w_{1} / w_{2}$ (by enlarging the capacitor length $l_{1}$ also achieves an electrical size reduction). Under these conditions, it is expected that the model is valid in a wider frequency range. We have performed the full-wave electromagnetic simulation of the structure for the differential mode, and we have extracted the elements of that model by means of a procedure reported elsewhere [10]. Qualitatively, that technique is based on measurable characteristics of the transmission and reflection coefficients provided by the electromagnetic simulation. Briefly, the lumped elements are obtained by means of the following conditions: (i) the transmission zero frequency corresponds to the frequency that the shunt impedance nulls, (ii) the frequency for which the shunt admittance vanishes is the resonant frequency of the parallel tank, (iii) the frequency whose phase of the transmission coefficient is $\pi / 2$ is where the series and the shunt impedances are conjugate, and (iv) the intersection between the reflection coefficient and the unit normalized resistance circle gives the series impedance. The electromagnetic simulation and the circuit simulation of the equivalent circuit model using the extracted parameters are depicted in Figure 7(b). As can be seen the agreement is good, hence validating the proposed model of the MLC-loaded differential microstrip line.

3.2. Single-Ended Microstrip Lines Loaded with MLCs. Let us now consider the MLC loading a single-ended microstrip transmission line, as depicted in Figure 8. Since the symmetry plane is a magnetic wall, the MLC cannot be excited. The proposed circuit model is depicted in Figure 9(a), which is similar to that reported in [11] for folded SIR-loaded coplanar waveguides. After applying the magnetic wall concept, this circuit model can be simplified as Figure 9(b) illustrates; 

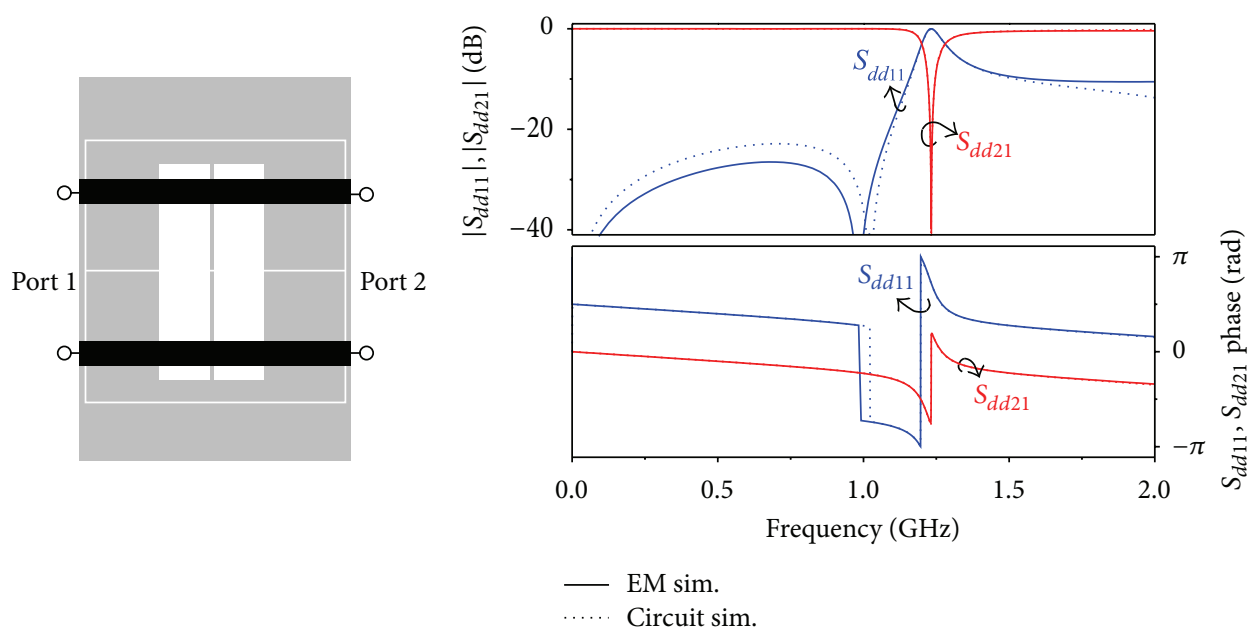

(a)

(b)

FIGURE 7: Layout (a) and differential mode frequency response (b) for a differential microstrip line loaded with an MLC with the electric wall aligned with the line and for its circuit model of Figure 6. The dimensions and the substrate are the same as those given in the caption of Figure 5, except for $l_{1}=2 \mathrm{~mm}$ and $w_{1}=8 \mathrm{~mm}$. The circuit parameters are $L=6.07 \mathrm{nH}, C=1.53 \mathrm{pF}, 4 C_{m}+C_{g} / 2=7.47 \mathrm{pF}$ (where for dimensions $C_{g} \approx 14 C_{m}$ ), and $L_{m}=3.71 \mathrm{nH}$.

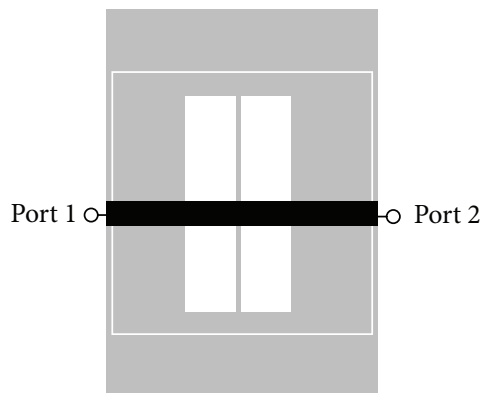

FIGURE 8: Microstrip line loaded with an MLC with the electric wall aligned with the line. The dimensions and the substrate are the same as those considered in Figure 7.

that is, it is a transmission line model with modified shunt capacitance. However, a notch in the transmission coefficient is not expected according to this model.

The interest in these single-ended microstrip transmission lines loaded with MLCs may be the truncation of symmetry, for instance, by means of a lateral displacement of the particle. In this case, the symmetry planes are no longer aligned, and the particle can be excited by the electric field generated by the line. The effect of this symmetry truncation by laterally shifting the MLC can be taken into account in the model of Figure 9(a) by simply considering the coupling capacitances (i.e., $C_{1}$ and $C_{2}$ ) as variable capacitances. From the circuit model viewpoint, these asymmetric values of the coupling capacitances allow current flowing through the MLC inductance and capacitance, and hence the particle is electrically driven by the line. As reported in [11], such kind of circuit can be useful for the implementation of displacement sensors or radio frequency (RF) barcodes.
We have simulated the structure of Figure 8 by considering two different lateral displacements $(0.5$ and $2 \mathrm{~mm})$ of the MLC. The results are depicted in Figure 10. In order to extract the lumped element values of its circuit model, as a starting point we consider the values given in the caption of Figure 7 since the same dimensions and substrate are considered. Afterwards, we have adjusted the variable coupling capacitances by curve fitting the electromagnetic simulation, which depend on the amount of displacement. The other elements of the circuit left nearly unaltered (a slight optimization may be required), except for the line inductance which is also modified by the position of the slotted resonator. The circuit simulations, also depicted in Figure 10, are in good agreement with the electromagnetic simulations. Therefore, the proposed model is validated, and it is demonstrated that the lateral shift of the MLC can be basically taken into account by modifying the coupling capacitances and the line inductance.

\section{Potential Applications}

Etched in the ground plane of differential microstrip lines, MLC resonators can be used for the implementation of balanced notch filters and stop-band filters. This functionality is clear to the light of the results depicted in Figure 7, where a notch for the differential mode appears in the vicinity of the fundamental resonance of the MLC. The rejection band can be widened by etching further MLC resonators with slightly different resonance frequency (as reported in [12] for the design of SRR-based CPW stopband filters) or with identical resonance frequency but separated by a small distance in order to enhance interresonator's coupling (as discussed in [13] in reference to common mode suppression in differential lines by means of CSRRs). 


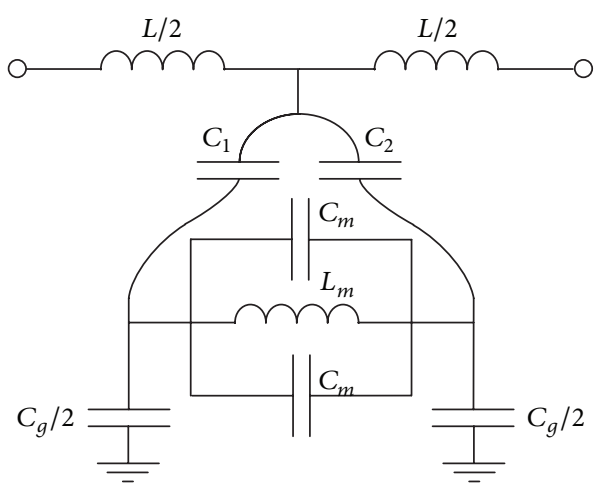

(a)

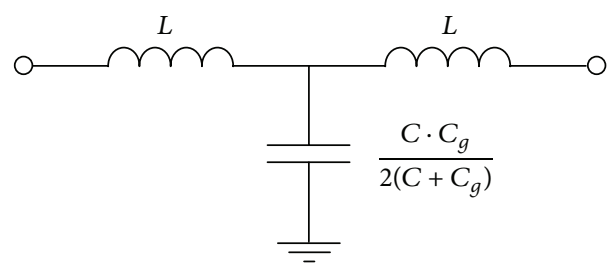

(b)

Figure 9: (a) Lumped element-equivalent circuit model of a microstrip line loaded with an MLC whose electric wall is aligned with or laterally displaced with regard to the line; (b) circuit model after applying the magnetic wall concept under the assumption of alignment, that is, $C_{1}=C_{2}=C / 2$.

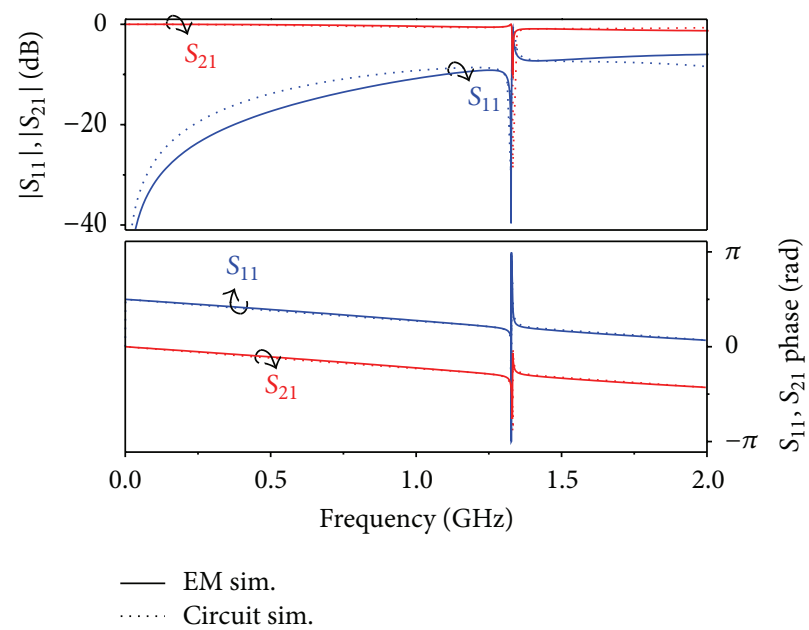

(a)

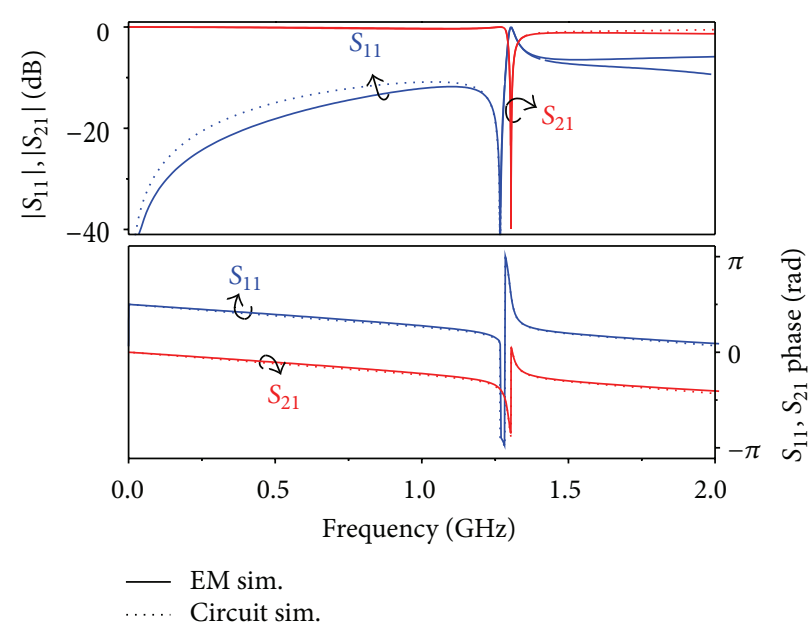

(b)

FIGURE 10: Frequency response for the structure of Figure 8 that results by laterally shifting the MLC $0.5 \mathrm{~mm}$ (a) and $2 \mathrm{~mm}$ (b) and for its circuit model of Figure 9. The circuit parameters are $L_{m}=3.71 \mathrm{nH}$ and $C_{g}=9.5 \mathrm{pF}$; for $0.5 \mathrm{~mm}, L=9.48 \mathrm{nH}, C_{1}=0.89 \mathrm{pF}, C_{2}=0.36 \mathrm{pF}$, and $C_{m}=0.59 \mathrm{pF}$; for $2 \mathrm{~mm}, L=8.94 \mathrm{nH}, C_{1}=1.36 \mathrm{pF}, C_{2}=0.083 \mathrm{pF}$, and $C_{m}=0.66 \mathrm{pF}$.

With regard to microstrip lines loaded with MLCs, we can take benefit of the effects of a lateral shift (or another means of symmetry truncation) in the transmission coefficient. Namely, the line is transparent if the electric wall of the MLC is perfectly aligned with the symmetry plane of the line. However, a notch appears when the MLC is shifted in the transverse direction, and the bandwidth and magnitude of the notch increase with the displacement (see Figure 10). Similarly, a notch is expected if the MLC is not displaced, but it is nonsymmetrically loaded with a certain dielectric load. Thus, the structure can be used for sensing purposes [14-17]. Furthermore, microstrip lines loaded with multiple MLCs can be used as RF bar codes, where each bit is associated to a certain resonator (and its resonance frequency), and the logic states "1" or " 0 " are simply set by etching the MLCs symmetrically or laterally shifted or by other means of truncating the symmetry (RF bar codes not based on such a symmetry approach were reported in [18]). As an example, Figure 11(a) shows a photograph of a 3-bit bar code with the central MLC aligned with the line and the external ones laterally displaced (corresponding to the code "101"). The frequency response is depicted in Figure 11(b) and exhibits a transmission zero at the frequencies of the external resonators, in coherence with the considered codification. The resonators were designed to achieve relatively narrow notches, thus diminishing interference between adjacent notches (this is a key issue to bits embedding and the related spectral band efficiency). Even though the notch level (intimately related to the bandwidth) is not very deep, this is not a critical drawback in binary bar codes. It is also interesting to mention that compared to CPW-based RF-bar codes, implemented by means of folded SIRs or SRRs (they also exhibit an electric wall at their symmetry plane) [11], the proposed bar code is more simple since no parasitic modes 


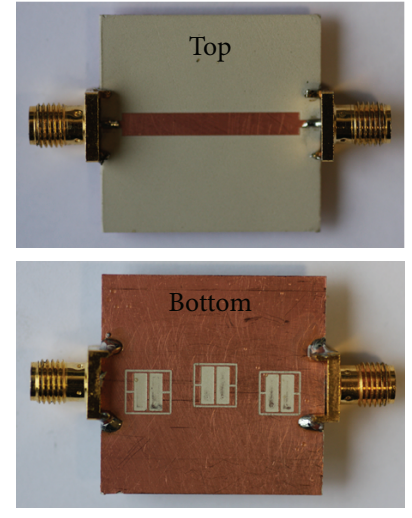

(a)

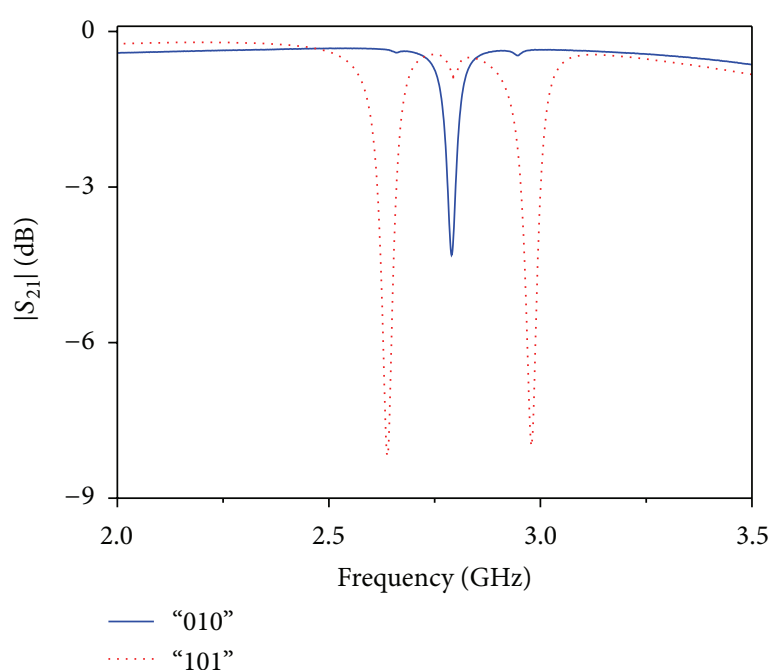

(b)

FIGURE 11: Photograph of a 3-bit RF bar code (codified as "101") (a) and measured frequency response (b). For completeness, the measurement for the code " 010 " is also shown. The substrate is Rogers $R O 3010$ with thickness $h=1.27 \mathrm{~mm}$, dielectric constant $\varepsilon_{r}=11.2$, and loss tangent $\tan \delta=0.0023$. The line width is designed to minimize the insertion loss with the presence of the MLCs symmetrically etched, that is, $W=3.13 \mathrm{~mm}$. The MLC dimensions are $w_{1}=5.2 \mathrm{~mm}, w_{2}=s=0.2 \mathrm{~mm}, l_{1}=1.9 \mathrm{~mm}, l_{2}(2.6 \mathrm{GHz})=6.32 \mathrm{~mm}, l_{2}(2.8 \mathrm{GHz})=6 \mathrm{~mm}$, $l_{2}(3 \mathrm{GHz})=5.72 \mathrm{~mm}$, and $l_{3}=6 \mathrm{~mm}$. The distance between adjacent resonators is set to that for which interresonator coupling can be neglected, that is, $3 \mathrm{~mm}$. The lateral displacement to set the logic state " 1 " is $1 \mathrm{~mm}$.

are generated by displacing the MLCs (in contrast, air bridges were necessary in the CPW bar codes reported in [11]). Alternatively, codification can be achieved by symmetrically etching the MLCs and truncating symmetry in the required resonator by adding adhesives with metallic or dielectric loads.

\section{Conclusions}

In conclusion, it has been shown that the MLC is a slot resonator that exhibits two symmetry planes, one being a magnetic wall and the other one an electric wall at the fundamental resonance. Given that other related slot resonators do not exhibit any electric wall, this paper focuses on such wall, which brings a wider variety of applications. If the driving fields of the particle are uniform, the MLC cannot be excited by an electric field orthogonal to the particle plane, but by means of a magnetic field in the plane of the particle. However, it has been shown that, in single-ended microstrip lines and differential microstrip lines, where the fields are no longer uniform, the MLC can be electrically excited. Equivalent circuit models of both differential and single-ended microstrip lines loaded with MLCs with the electric wall aligned with the symmetry plane of the lines have been reported and validated. Finally, some applications have been highlighted, including balanced notch filters, as well as sensors and bar codes based on the truncation of symmetry.

\section{Acknowledgments}

This work has been supported by MINECO, Spain (Projects TEC2010-17512, CSD2008-00066, and TEC2011-13615-E) and by AGAUR-Generalitat de Catalunya, through Project 2009SGR-421. Jordi Naqui is also in debt to MINECO Spain for supporting his work through the FPU Grant AP20100431.

\section{References}

[1] J. B. Pendry, A. J. Holden, D. J. Robbins, and W. J. Stewart, "Magnetism from conductors and enhanced nonlinear phenomena," IEEE Transactions on Microwave Theory and Techniques, vol. 47, no. 11, pp. 2075-2084, 1999.

[2] F. Martín, J. Bonache, F. Falcone, M. Sorolla, and R. Marqués, "Split ring resonator-based left-handed coplanar waveguide," Applied Physics Letters, vol. 83, no. 22, pp. 4652-4654, 2003.

[3] F. Falcone, T. Lopetegi, J. D. Baena, R. Marqués, F. Martín, and M. Sorolla, "Effective negative- $\varepsilon$ stopband microstrip lines based on complementary split ring resonators," IEEE Microwave and Wireless Components Letters, vol. 14, no. 6, pp. 280-282, 2004.

[4] R. Marqués, F. Martín, and M. Sorolla, Metamaterials with Negative Parameters: Theory, Design and Microwave Applications, John Wiley \& Sons, New York, NY, USA, 2008.

[5] R. Marques, F. Medina, and R. Rafii-El-Idrissi, "Role of bianisotropy in negative permeability and left handed metamaterials," Physical Review B, vol. 65, paper 144441, 2002.

[6] J. D. Baena, J. Bonache, F. Martín et al., "Equivalent-circuit models for split-ring resonators and complementary splitring resonators coupled to planar transmission lines," IEEE Transactions on Microwave Theory and Techniques, vol. 53, no. 4, pp. 1451-1460, 2005.

[7] D. R. Smith, W. J. Padilla, D. C. Vier, S. C. Nemat-Nasser, and S. Schultz, "Composite medium with simultaneously negative 
permeability and permittivity," Physical Review Letters, vol. 84, no. 18, pp. 4184-4187, 2000.

[8] F. Falcone, T. Lopetegi, M. A. G. Laso et al., "Babinet principle applied to the design of metasurfaces and metamaterials," Physical Review Letters, vol. 93, no. 19, Article ID 197401, 2004.

[9] D. Schurig, J. J. Mock, and D. R. Smith, "Electric-field-coupled resonators for negative permittivity metamaterials," Applied Physics Letters, vol. 88, no. 4, Article ID 041109, pp. 1-3, 2006.

[10] J. Bonache, M. Gil, I. Gil, J. Garcia-García, and F. Martín, “On the electrical characteristics of complementary metamaterial resonators," IEEE Microwave and Wireless Components Letters, vol. 16, pp. 543-545, 2006.

[11] J. Naqui, M. Durán-Sindreu, and F. Martín, "On the symmetry properties of coplanar waveguides loaded with symmetric resonators: analysis and potential applications," in Proceedings of IEEE MTT-S International Microwave Symposium, Montreal, Canada, June 2012.

[12] F. Martín, F. Falcone, J. Bonache, R. Marqués, and M. Sorolla, "Miniaturized coplanar waveguide stop band filters based on multiple tuned split ring resonators," IEEE Microwave and Wireless Components Letters, vol. 13, no. 12, pp. 511-513, 2003.

[13] J. Naqui, A. Fernández-Prieto, M. Durán-Sindreu et al., "Common mode suppression in microstrip differential lines by means of complementary split ring resonators: theory and applications," IEEE Transactions on Microwave Theory and Techniques, vol. 60, pp. 3023-3034, 2012.

[14] C. Mandel, B. Kubina, M. Schusler, and R. Jakoby, "Passive chipless wireless sensor for two-dimensional displacement measurement," in Proceedings of the 14th European Microwave Conference (EuMC '11), pp. 79-82, October 2011.

[15] J. Naqui, M. Durán-Sindreu, and F. Martín, "Alignment and position sensors based on split ring resonators," Sensors, vol. 12, pp. 11790-11797, 2012.

[16] M. Puentes, M. Maasch, M. Schubler, and R. Jakoby, "Frequency multiplexed 2-dimensional sensor array based on split-ring resonators for organic tissue analysis," IEEE Transactions on Microwave Theory and Techniques, vol. 60, no. 6, pp. 1720-1727, 2012.

[17] A. Karami Horestani, C. Fumeaux, S. F. Al-Sarawi, and D. Abbott, "Displacement sensor based on diamond-shaped tapered split ring resonator," IEEE Sensors Journal, vol. 13, no. 4, pp. 1153-1160, 2013.

[18] S. Preradovic and N. C. Karmakar, "Chipless RFID: bar code of the future," IEEE Microwave Magazine, vol. 11, no. 7, pp. 87-97, 2010 . 

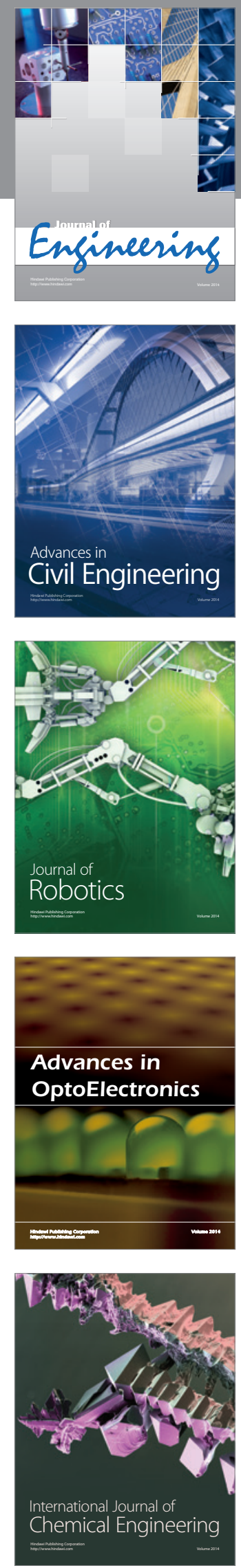

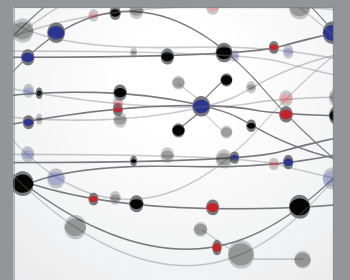

The Scientific World Journal
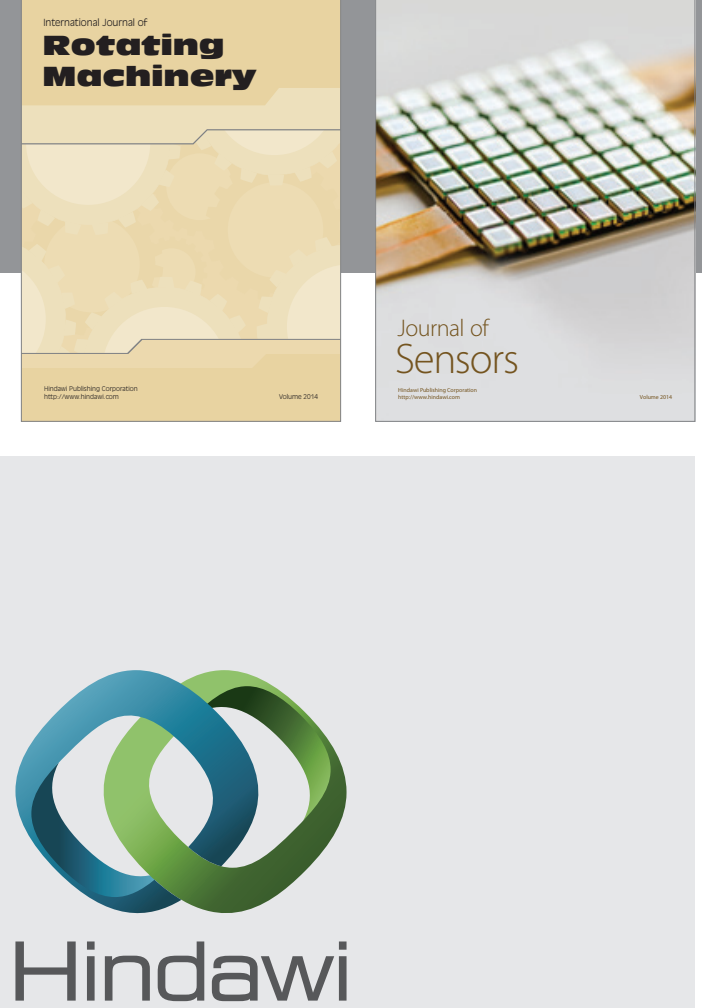

Submit your manuscripts at http://www.hindawi.com
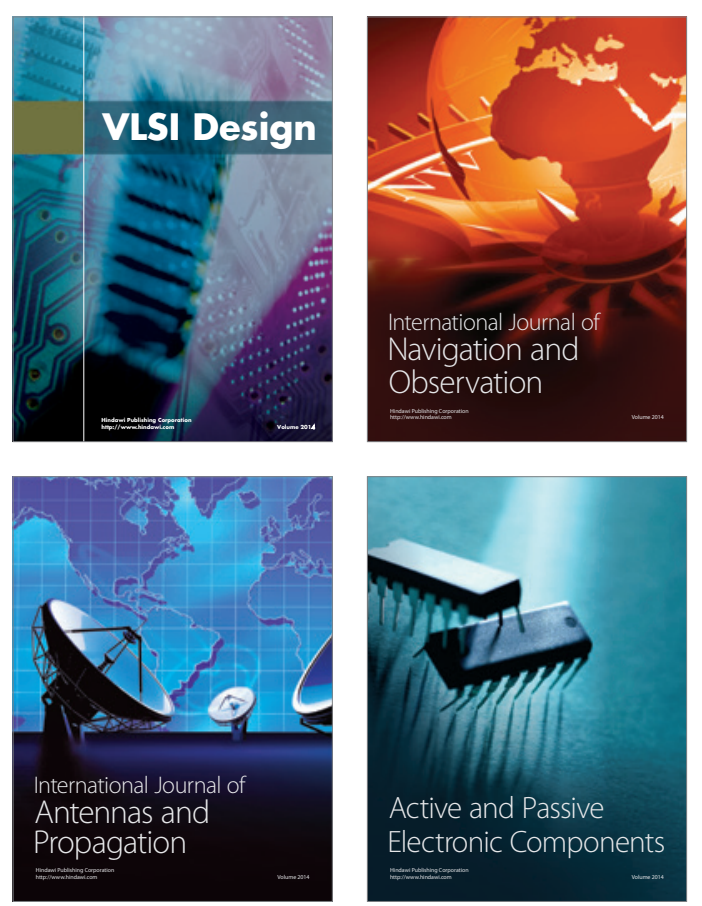
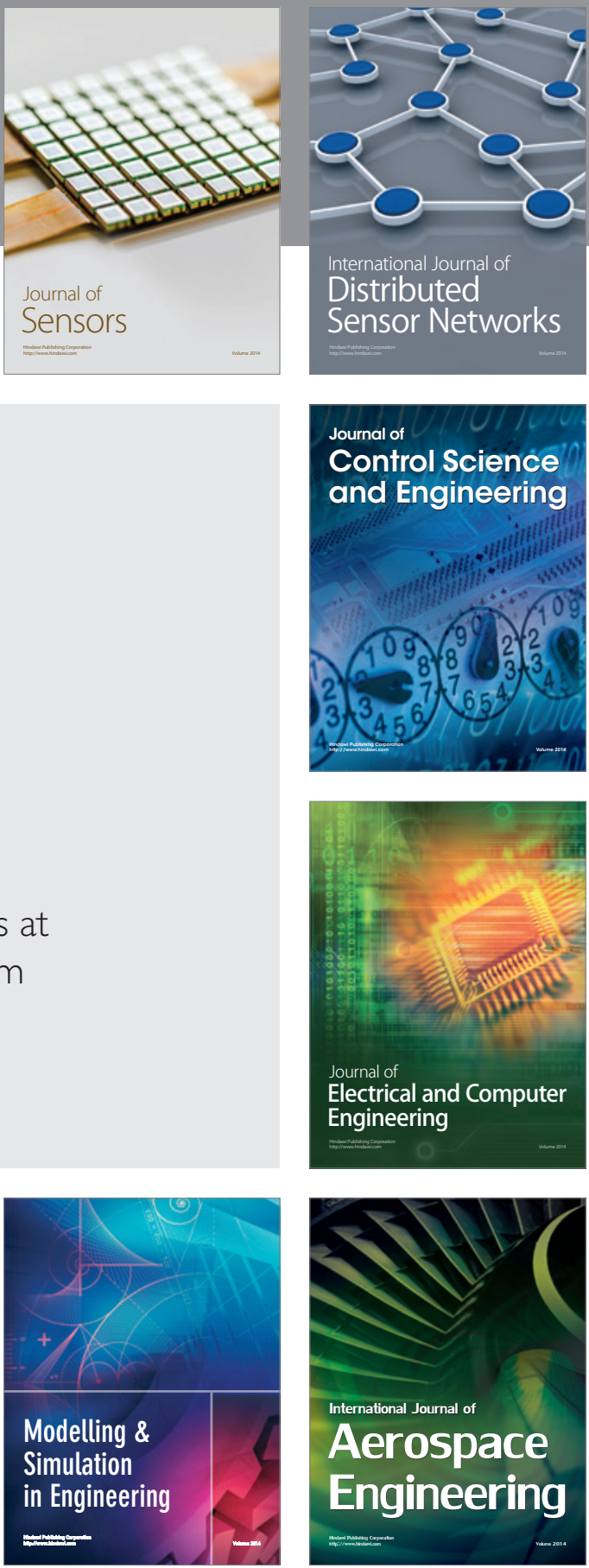

Journal of

Control Science

and Engineering
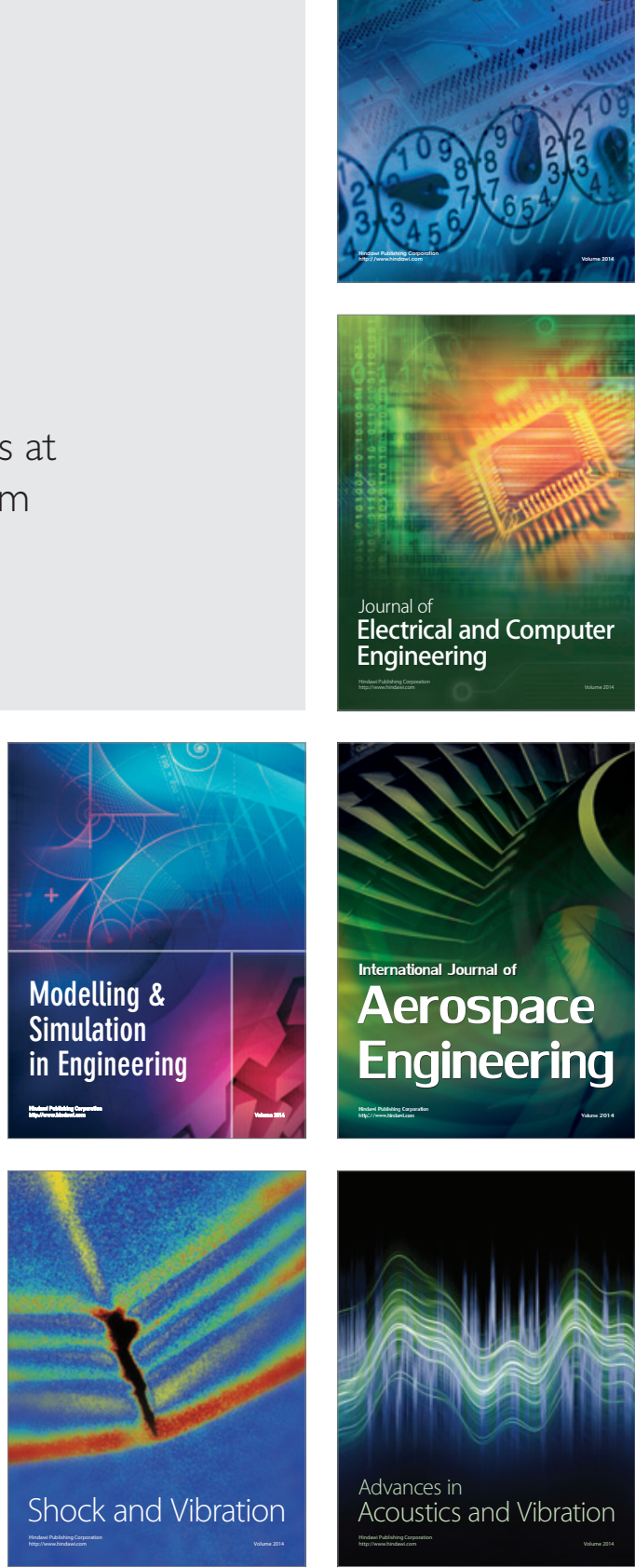